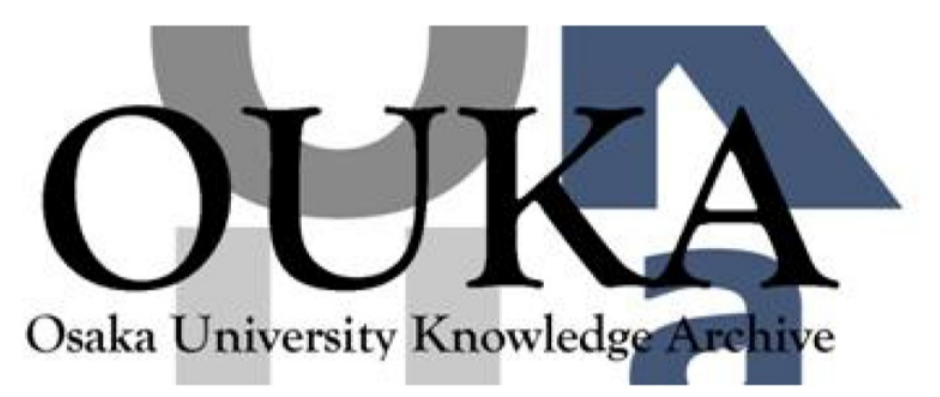

\begin{tabular}{|c|l|}
\hline Title & $\begin{array}{l}\text { Side-on measurement of hydrodynamics of laser- } \\
\text { driven plasmas with high space- and time- } \\
\text { resolution x-ray imaging technique }\end{array}$ \\
\hline Author(s) & $\begin{array}{l}\text { Fujioka, Shinsuke; Shiraga, Hiroyuki; } \\
\text { Nishikino, Massaharu et al. }\end{array}$ \\
\hline Citation & $\begin{array}{l}\text { Review of Scientific Instruments. 74(3) p. 2198- } \\
\text { p. } 2201\end{array}$ \\
\hline Issue Date & $2003-03$ \\
\hline oaire:version & VoR \\
\hline URL & https://hdl. handle. net/11094/2879 \\
\hline rights & \\
\hline Note & \\
\hline
\end{tabular}

Osaka University Knowledge Archive : OUKA

https://ir. Library. osaka-u. ac. jp/

Osaka University 


\title{
Side-on measurement of hydrodynamics of laser-driven plasmas with high space- and time-resolution x-ray imaging technique
}

\author{
Shinsuke Fujioka ${ }^{a}$ \\ Institute of Laser Engineering, Osaka University, 2-6 Yamada-oka, Suita Osaka 565-0871, Japan and \\ Department of Electro, Information System, and Energy Engineering, Osaka University, Suita Osaka $565-$ \\ 0871, Japan \\ Hiroyuki Shiraga, Masaharu Nishikino, Yohei Tamari, Keisuke Shigemori, Mitsuo Nakai, \\ and Hiroshi Azechi \\ Institute of Laser Engineering, Osaka University, 2-6 Yamada-oka, Suita Osaka 565-0871, Japan \\ Kazuo A. Tanaka \\ Institute of Laser Engineering, Osaka University, 2-6 Yamada-oka, Suita Osaka 565-0871, Japan and \\ Department of Electro, Information System, and Energy Engineering, Osaka University, Suita Osaka 565- \\ 0871, Japan \\ Tatsuhiko Yamanaka \\ Institute of Laser Engineering, Osaka University, 2-6 Yamada-oka, Suita Osaka 565-0871, Japan
}

(Presented on 11 July 2002)

\begin{abstract}
We developed and improved high space- and time-resolution x-ray imaging techniques $(3-6 \mu \mathrm{m}$, 100-200 ps). These techniques were coupled with side-on x-ray backlighting for observing hydrodynamics of directly laser-driven polystyrene plasmas. As a high sensitive x-ray image detector, Imaging Plate was introduced to penumbral imaging technique [S. Fujioka et al., Rev. Sci. Instrum. 73, 2588 (2002)]. Noise in penumbral images was reduced by this improvement, and high quality density profiles of plasmas were obtained. A large-magnification slit camera, whose slit width and magnification were $4 \mu \mathrm{m}$ and 82.8 , was coupled with an x-ray streak camera (XSC). Due to the advantage of the large-magnification, high spatial resolution of $5.8 \mu \mathrm{m}$ was found even with an XSC, the photocathode of which usually has low spatial resolution. These techniques are useful in understanding of hydrodynamic instability in inertial confinement fusion. (C) 2003 American Institute of Physics. [DOI: 10.1063/1.1537852]
\end{abstract}

\section{INTRODUCTION}

In the scenario of directly laser-driven inertial confinement fusion (ICF), ${ }^{1}$ a target shell implodes inwardly due to laser ablation. Through the implosion, the dense shell is adjacent to a light corona plasma with an effective outwardly directed gravitational force. This is the standard situation for occurrence of the Rayleigh-Taylor (RT) instability. Fortunately, on the outer surface of the ICF target, growth of the RT instability is stabilized by the laser ablation..$^{2-4}$ This is called generally the ablative stabilization. For achieving a high gain ICF, we need to understand the stabilization mechanism and enhance the stabilization effect.

The density structure in the laser-driven plasmas is one of the most important objectives for understanding of the ablative stabilization because the RT growth depends on the density structure, i.e., the peak density and the minimum scale length of density gradient. Recently, side-on backlighting coupled with the high space- and time-resolution $\mathrm{x}$-ray imaging technique was developed to observe the detailed hydrodynamics including the density structure of the laser-driven plasmas. ${ }^{5}$ The measured hydrodynamics gives us the basic physical knowledge on the growth and the stabilization of the RT instability.

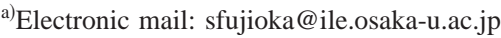

In this article we present experimental results obtained with $\mathrm{x}$-ray penumbral imaging ${ }^{6}$ and a large-magnification slit camera. The penumbral imaging was improved by using a high sensitive x-ray image detector of the imaging plate (IP, Fuji Film Co. Ltd.). ${ }^{7}$ As a result of this improvement, noise in the penumbral images was reduced clearly, and high quality density profiles were obtained. The large-magnification slit camera was coupled with an x-ray streak camera (XSC). Due to an advantage of the large magnification, high spatially resolved and also temporal sequential x-ray radiographs of plasmas were obtained with this technique.

\section{MEASUREMENT OF LASER-DRIVEN PLASMAS WITH SIDE-ON X-RAY BACKLIGHTING}

When a plasma is probed by $\mathrm{x}$ rays along the path parallel to the target surface, an $\mathrm{x}$-ray radiograph of the plasma is obtained with the $\mathrm{x}$-ray imaging technique. The $\mathrm{x}$-ray radiograph possesses information on the density profile of the plasma. The $\mathrm{x}$-ray transmission $[T(x)]$ of the plasma is determined by the length of the $\mathrm{x}$-ray path through the plasma $(l)$, the mass absorption coefficient $[\mu(x)]$, and the density $[\rho(x)]$ of the target material as $T(x)=\exp [-\mu(h \nu) \rho(x) l]$, where $x$ denotes the position in the coordinate along the density gradient (normal to the target surface), $h$ and $\nu$ are Planck's constant and the frequency of the probe $\mathrm{x}$ ray. In the 


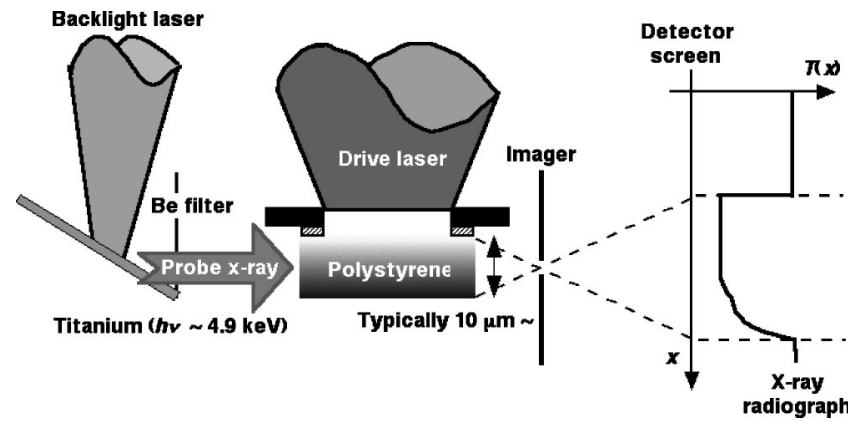

FIG. 1. A sketch of side-on x-ray backlighting for observing hydrodynamics of laser-driven polystyrene plasmas.

experiment, we used a polystyrene $\left(\mathrm{PS}, \mathrm{C}_{8} \mathrm{H}_{8}\right)$ foil whose width is $200 \mu \mathrm{m}$ as the drive target and a titanium (Ti) plasma as the backlight x-ray source (see Fig. 1). The x-ray transmission of the backlight $\mathrm{x}$ ray depending on areal density of the PS was calibrated, the dependence was found to be almost equal to the tabulated one at $4.85 \mathrm{keV}$ for neutral PS. ${ }^{5}$

A typical spatial scale of laser-driven PS plasma is about several tens of micrometers, and its typical velocity is about $30-50 \mathrm{~km} / \mathrm{s}\left[=(0.3-0.5) \times 10^{7} \mathrm{~cm} / \mathrm{s}\right]$ in our experimental condition. The high space- and also time-resolution x-ray imaging technique is required for observing the structure in detail. We developed two types of $\mathrm{x}$-ray imaging techniques, whose spatial resolution is 3-6 $\mu \mathrm{m}$ and temporal resolution is $100-200$ ps. One is the penumbral imaging coupled with flash x-ray backlighting and an IP, and the other is the largemagnification slit camera coupled with an XSC.

\section{EXPERIMENTAL CONDITION}

We have performed the experiment with the GEKKO XII-HIPER (High Intensity Plasma Experimental Research) laser facility. ${ }^{8}$ In the HIPER system, nine beams consisting of a frequency-tripled $\left(\lambda_{L}=0.35 \mu \mathrm{m}\right)$ laser smoothed by two-dimensional smoothing by spectral dispersion (2D-SSD $)^{9}$ were used for uniformly driving the PS targets. The other three beams consisting of a frequency-doubled $\left(\lambda_{L}=0.53 \mu \mathrm{m}\right)$ light were used as a foot pulse for precompression of the PS target, or used for generating backlight $\mathrm{x}$ ray in several shots. The beams were partially coherent light $(\mathrm{PCL})^{10}$ in the case of foot pulse, or coherent light in the case of generating backlight $\mathrm{x}$ ray. All beams were implemented with kinoform phase plates $(\mathrm{KPP})^{11}$ to obtain profilecontrolled uniform laser irradiation. When we used the foot pulse, the 13th frequency doubled $\left(\lambda_{L}=0.53 \mu \mathrm{m}\right)$ coherent beam implemented with a random phase plate $(\mathrm{RPP})^{12}$ was used for generating backlight $\mathrm{x}$ rays. Typical laser intensity of the main pulse was $I_{L}=0.7 \times 10^{14} \mathrm{~W} / \mathrm{cm}^{2}$ in a pulse duration of $2.5 \mathrm{~ns}$, and that of the foot pulse was $I_{L}=1.0$ $\times 10^{12} \mathrm{~W} / \mathrm{cm}^{2}$ in $2.0 \mathrm{~ns}$.

Low mode irradiation nonuniformity of the main drive laser was $\pm 5 \%$ (rms) within $200 \mu \mathrm{m}$ at the target center. This value was calculated from the spatial distribution of differences of shock breakout time obtained in a separate measurement with an S-20 streak camera. Therefore the bowing of the laser-driven target is less than $2-3 \mu \mathrm{m}$ (peakto-valley) in this experiment.

The laser-driven targets was a PS (initial density 1.06 $\mathrm{g} / \mathrm{cm}^{3}$ ) foil, whose initial thickness was 40 or $65 \mu \mathrm{m}$, and width was $200 \mu \mathrm{m}$ along the line of sight of $\mathrm{x}$-ray backlighting. The $\mathrm{x}$-ray backlight source was Ti plasmas irradiated by the laser beams which delivered $1 \mathrm{~kJ}$ in a $3.0 \mathrm{~ns}$ square pulse shape, or $100 \mathrm{~J}$ in a 100 ps Gaussian pulse shape. A 20- $\mu \mathrm{m}-$ thick Be foil was placed between the backlighter and the PS target to avoid preheating of the PS target due to soft x-ray emission from the backlight target.

\section{PENUMBRAL IMAGING COUPLED WITH IMAGING PLATE}

The penumbral imaging is one of the coded imaging techniques. The original image of an objective is reconstructed by differentiating a penumbra of the objective. High spatially resolved x-ray images can be obtained with the penumbral imaging technique, but it is intolerant to noise. The noise primarily degrades the quality of the reconstructed image, and may easily restrict the spatial resolution. We improved the penumbral imaging technique by introducing a high sensitive $x$-ray image detecter of the IP. The IP utilizes photostimulable phosphor of $\mathrm{BaFBr}: \mathrm{Eu}^{2+}$. While the IP has been used in wide scientific fields, for example, biology and material physics, there are a few cases in which the IP is introduced to laser-plasma experiments. We now purpose that the IP is a very useful x-ray image detector also for the laser-plasma experiment. Note that the IP has been used as an electron detecter for investigation of ultraintense laser and plasma interactions by a group at Osaka University in Japan. ${ }^{13}$

Compared with an x-ray charged coupled device (CCD), the IP has several advantages; (i) high x-ray detection quantum efficiency, (ii) large $\mathrm{x}$-ray sensitive area size, and (iii) wide dynamic range. These advantages are very suitable for reducing the noise in the penumbral image. Its disadvantages are (i) troublesome process of reading photostimulated light from the IP and (ii) large digitized pixel size. These are insignificant for our purpose or are able to be overcome.

The IP has a thick x-ray sensitive layer composed of relatively high- $Z$ material, and its detection quantum efficiency is as high as $100 \%$ for $x$ rays with energy less than 10 $\mathrm{keV}^{7}$ In x-ray images, statistical fluctuation in the number of the detected photon is the dominant source of noise. ${ }^{5}$ The noise in x-ray images can be reduced with using the IP due to increasing the number of the detected photon.

The $\mathrm{x}$-ray sensitive area size of the $\mathrm{x}$-ray CCD camera is typically less than $1 \times 1$ in. $^{2}$ square. On the other hand, the IP can have a very large $x$-ray sensitive area size of 200 $\times 250 \mathrm{~mm}^{2}$, and we used the IP cut into adequate size $(60$ $\times 60 \mathrm{~mm}^{2}$ ) in the experiment. This large sensitive area size brings out a merit in reducing the noise. In the case of onedimensional (1D) penumbral imaging by using a knife edge, the obtained image consists of many lines of 1D penumbral profile data. By using a large IP, the noise in the penumbral 

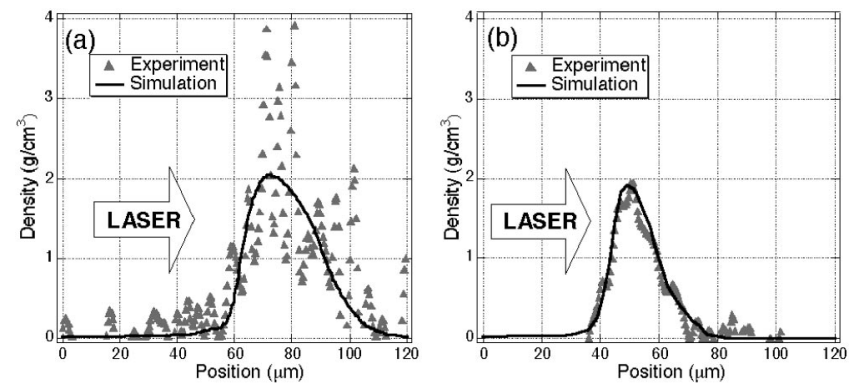

FIG. 2. (a) A density profile observed with the penumbral imaging and the x-ray CCD camera. (b) A density profile observed with the penumbral imaging and the imaging plate. Triangular points represent experimentally obtained density profiles, and solid lines represent postprocessed simulation results calculated with the $1 \mathrm{D}$ hydrodynamic simulation code. The quality of the density profile was improved clearly by introducing the imaging plate to the penumbral imaging.

profile can be reduced by averaging a lot of $1 \mathrm{D}$ penumbral data.

The digitized pixel size of the IP is $50 \times 50 \mu \mathrm{m}^{2}$ square in our IP reader, which is determined by the spot size from a $\mathrm{He}-\mathrm{Ne}$ laser in the reader. This size is larger than that of a generally used x-ray CCD camera (typically about $20 \mu \mathrm{m}$ ). We overcame this disadvantage by setting the penumbral imager as close as possible to an objective and enlarging the magnification of the imager. Setting the imager as close as possible to an objective has another advantage for the noise reduction, which increases the incident photon onto the detector per unit area of the objective plane. Fortunately, the large sensitive area size of the IP makes it easy to align such a large-magnification penumbral imager.

One of the most important characters which must be confirmed before the application is the linearity of the IP to pulse $\mathrm{x}$-ray originated from a laser-plasma. We measured the linearity with piled aluminum (Al) filters and wellcharacterized X-ray originated from a laser produced $\mathrm{Ti}$ plasma. Measured x-ray transmission depending on areal density of $\mathrm{Al}$ is consistent with tabulated transmission at 4.8 $\mathrm{keV}$ for neutral $\mathrm{Al}$, and the result indicates the sufficient linearity of the IP.

In the experiment, an edge polished $100-\mu \mathrm{m}$-thick tantalum plate was used as the penumbral imager. We set the imager at $p=30 \mathrm{~mm}$ [Fig. 2(a)] or $p=15 \mathrm{~mm}$ [Fig. 2(b)] away from the target, and at $f=1575 \mathrm{~mm}$ [Figs. 2(a) and 2(b) ] away from the X-ray image detector, so that its magnification $(M=f / p)$ was 51 [Fig. 2(a)] or 105 [Fig. 2(b)]. The IP and x-ray CCD camera themselves do not have sufficient temporal resolution, flash $\mathrm{x}$-ray backlighting was used for observing instantaneous density profiles in laser-driven plasmas.

Figures 2(a) and 2(b) show the density profiles obtained with the X-ray CCD camera and the IP, respectively. In Fig. 2 , the origin of the position was set to be the initial position of the target front surface. These density profiles were reconstructed using the same Wiener-filter and the same spatial separation $(1.8 \mu \mathrm{m}$ on target) in the differentiation process. Initial thickness of the PS foil is $65 \mu \mathrm{m}$ [Fig. 2(a)] and 40 $\mu \mathrm{m}$ [Fig. 2(b)]. The time of backlight x-ray flashing was $0.8 \pm 0.1 \mathrm{~ns}$ and $0.6 \pm 0.1 \mathrm{~ns}$ after the shock break out at the target rear surface. The duration of flash $\mathrm{X}$-ray pulse was measured with an X-ray streak camera (XSC) with temporal resolution of $10 \mathrm{ps}$. It was in a nearly Gaussian shape, whose full width at half maximum (FWHM) was 160 ps [Fig. 2(a)] and 140 ps [Fig. 2(b)]. Figure 2 shows also simulation results calculated by the 1D hydrodynamic simulation code (ILESTA-1D). ${ }^{14}$ These simulation results were postprocessed taking into account motion blurring of the target and the response function of the Wiener filter used in the reconstruction process. As shown clearly in the comparison, the density profile obtained with the IP has a higher signal-to-noise $(\mathrm{S} / \mathrm{N})$ ratio than that obtained with the $\mathrm{x}$-ray $\mathrm{CCD}$ camera. This improvement is due to the high detection quantum efficiency of the IP, the large magnification of the penumbral imager, and averaging a lot of penumbral data obtained with the large IP. This result indicates that the IP is a more suitable x-ray image detector than an x-ray CCD camera.

\section{LARGE-MAGNIFICATION SLIT CAMERA COUPLED WITH X-RAY STREAK CAMERA}

Slit camera imaging is a conventional 1D imaging technique widely used in the laser-plasma research. Spatial resolution $(\Delta r)$ of a slit camera is determined by a geometrical contribution by the finite slit width and a diffractive contribution: $\Delta r=\sqrt{[D(1+1 / M)]^{2}+(2.0 \cdot \lambda / D \cdot p)^{2}}$, where $M, D$, $\lambda$, and $p$ represent magnification, slit width, wavelength of incident photon, and distance between a slit and an objective, respectively. ${ }^{15}$ When the slit is set close to the objective, the diffractive contribution to the spatial resolution becomes small. Furthermore, the magnification of the slit camera becomes large because of the small distance between the objective and the slit. Due to the advantage of the large magnification, we can obtain high spatially resolved x-ray images with an XSC, the photocathode of which has a low spatial resolution (typically $200 \mu \mathrm{m}$ ).

The slit used in this experiment is $4 \times 200 \mu \mathrm{m}^{2}$. The slit was set at $p=17 \mathrm{~mm}$ away from the target and at $f$ $=1408 \mathrm{~mm}$ away from the XSC. Magnification of the slit camera was $M=82.8$. We used a $1100-\AA$-thick CuI coated on a parylene substrate as a photocathode of the XSC. The slit width of the photocathode was $350 \mu \mathrm{m}$ in the sweep direction. This width corresponds to the temporal resolution of 200 ps. Spatial resolution of $5.8 \mu \mathrm{m}$ was found from the $\mathrm{x}$-ray backlit image of a tantalum wire.

In the experiment, a trajectory of the laser-driven PS target were observed with the large-magnification slit camera coupled with the XSC. Unfortunately, we could not obtain high quality $\mathrm{x}$-ray radiographs of the PS targets with this imaging technique due to a low sensitivity of the XSC and a low intensity of the backlight $\mathrm{x}$ rays. However, we still can compare the trajectories of shock front, positions of peak density, and fluids whose density are equal to $0.5 \mathrm{~g} / \mathrm{cm}^{3}$ at front and rear target surfaces between the experiment and the simulation. The reason why we choose the density of 0.5 $\mathrm{g} / \mathrm{cm}^{3}$ in the comparison is that a change of a transmission in proportion to a change of a density $\left(\partial T / \partial \rho=-\rho e^{-\rho}\right)$ is large in the vicinity of $0.5 \mathrm{~g} / \mathrm{cm}^{3}$, and the density calculation is expected to be precise. 

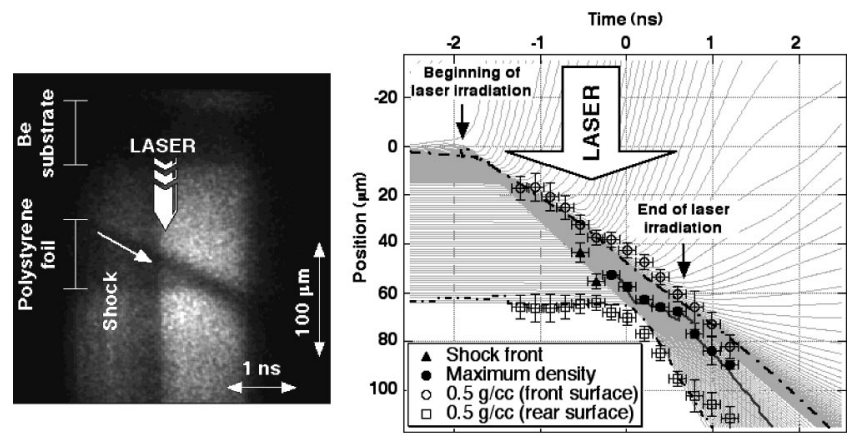

(a)

(b)
FIG. 3. (a) An x-ray radiograph of the laser-driven polystyrene plasma observed with the large-magnification slit camera coupled with the x-ray streak camera. (b) Trajectories of the plasma obtained in the experiment and calculated with the 1D hydrodynamic simulation code. Broken lines represent simulated trajectories of fluids whose densities are $0.5 \mathrm{~g} / \mathrm{cm}^{3}$ at the front and rear surface, respectively. The solid line represents the simulated trajectory of positions of the peak density. Marks of open circle, open square, closed circle, and closed triangle represent experimentally obtained trajectories of fluids whose densities are $0.5 \mathrm{~g} / \mathrm{cm}^{3}$ at the front and rear surface, positions of the peak density, and shock front, respectively.

Initial thickness of the laser-driven target was $65 \mu \mathrm{m}$. The foot pulse was not irradiated in this experiment. Figure 3(a) shows the radiograph of the laser-driven polystyrene target. Figure 3(b) shows the experimentally obtained trajectory and the simulation result calculated by the ILESTA-1D code. In Fig. 3(b), the origin of time is the time when shock has broken out at the target rear surface, and the origin of position was the position of the initial target surface. This result indicates that the ILESTA-1D code precisely predicts the gross hydrodynamics of the laser-driven PS targets (velocity, acceleration, and shock velocity) as long as laser drive was on.

At the present time, we could not obtain a high quality $\mathrm{x}$-ray radiograph of the laser-driven PS plasma with this imaging technique. However, we believe that a clear temporal evolution of the hydrodynamics including the density profile can be obtained with this technique, when high intensity backlight $\mathrm{x}$ ray is available.

\section{ACKNOWLEDGMENTS}

The authors would like to thank the staff at the Institute of Laser Engineering for technical assistance in the operation of the GEKKO XII-HIPER laser system, target fabrication, and plasma diagnostics. One of the authors (S. F.) gratefully acknowledges the financial support from the Hiroshi Kondo scholarship of Osaka University.

${ }^{1}$ J. H. Nuckolls, L. Wood, A. Thiessen, and G. B. Zimmerman, Nature (London) 239, 139 (1972).

${ }^{2}$ S. E. Bodner, Phys. Rev. Lett. 33, 761 (1974).

${ }^{3}$ H. Takabe, K. Mima, L. Montierth, and R. L. Morse, Phys. Fluids 28, 3679 (1985).

${ }^{4}$ R. Betti, V. N. Goncharov, R. L. McCrory, and C. P. Verdon, Phys. Plasmas 5, 1446 (1998).

${ }^{5}$ S. Fujioka, H. Shiraga, M. Nishikino, M. Heya, K. Shigemori, M. Nakai, H. Azechi, S. Nakai, and T. Yamanaka, Rev. Sci. Instrum. 73, 2588 (2002).

${ }^{6}$ K. A. Nugent and B. Luther-Davies, Opt. Commun. 49, 393 (1984).

${ }^{7}$ M. Ito and Y. Amemiya, Nucl. Instrum. Methods Phys. Res. A 310, 369 (1991).

${ }^{8}$ N. Miyanaga, M. Nakatsuka, H. Azechi, H. Shiraga, T. Kanabe, H. Asahara, H. Daido, H. Fujita, and K. F. K. Fujita, Proceedings of the 18th International Conference on Fusion Energy (IAEA, Sorrento, Italy, 2001), IAEA-CN-77.

${ }^{9}$ S. Skupsky, R. W. Short, T. Kessler, R. S. Craxton, S. Letzring, and J. M. Soures, J. Appl. Phys. 66, 3456 (1989).

${ }^{10}$ H. Nakano, N. Miyanaga, K. Yagi, K. Tsubakimoto, T. Kanabe, M. Nakatsuka, and S. Nakai, Appl. Phys. Lett. 63, 580 (1993).

${ }^{11}$ S. N. Dixit, J. K. Lawson, K. R. Manes, H. T. Powell, and K. A. Nugent, Opt. Lett. 19, 417 (1994).

${ }^{12}$ Y. Kato, K. Mima, N. Miyanaga, S. Arinaga, Y. Kitagawa, M. Nakatsuka, and C. Yamanaka, Phys. Rev. Lett. 53, 1057 (1984).

${ }^{13}$ K. A. Tanaka, R. Kodama, H. Fujita, M. Heya, N. Izumi, Y. Kato, Y. Kitagawa, K. Mima, and N. M. T. Norimatsu, Phys. Plasmas 7, 2014 (2000).

${ }^{14}$ H. Takabe, M. Yamanaka, K. Mima, C. Yamanaka, H. Azechi, N. Miyanaga, M. Nakatsuka, T. Jitsuno, and T. N. M. Takagi, Phys. Fluids 10, 2884 (1988).

${ }^{15}$ J. A. Koch, O. L. Landen, T. W. Barbee, J. P. Celliers, L. B. D. Silva, S. G. Glendinning, B. A. Harmmel, D. H. Kalantar, and C. B. J. Seely, Appl. Opt. 37, 1784 (1998). 